\title{
Influence du pâturage sur la phytodiversité et la variation de la composition chimique de cinq plantes broutées dans le mont de Tessala (Algérie nord occidentale)
}

Kouider Cherifi ${ }^{1 *}$, Amar Bouker ${ }^{1}$, Mohammed Benabbou$^{1}$, Djillali Guemour ${ }^{2}$ et Okkacha Hasnaoui ${ }^{3}$

${ }^{1}$ Laboratoire de Biodiversité Végétale: Conservation et Valorisation, Université Djillali Liabès, BP 89, Haï Larbi Ben M'Hidi, Sidi Bel Abbés 22000, Algérie.

${ }^{2}$ Laboratoire d'Agrobiotechnologie et de Nutrition en Zones Semi-arides, Université Ibn Khaldoun, Tiaret, Algérie.

${ }^{3}$ Laboratoire d'Ecologie et de Gestion des Ecosystèmes Naturels, Université Abou-Bakr Belkaid, Tlemcen, Algérie.

\section{Correspondencia}

Kouider Cherifi

e-mail: cherifi.kouider@yahoo.com

Recibido: 8 febrero 2021

Aceptado: 3 mayo 2021

Publicado on-line: 6 julio 2021

Editado por: Noelia Hidalgo Triana

\begin{abstract}
Résumé
Le but de notre travail est d'évaluer l'impact du pâturage sur la biodiversité végétale des monts de Tessala. Pour cela, six (06) stations situées sur les versants nord et sud de la zone d'étude ont été échantillonnées. Sur chaque station une étude floristique et des analyses pédologiques sont effectuées afin de dégager les principaux facteurs qui interviennent dans la répartition de la végétation. En plus, des enquêtes sur terrain ont été menées afin de réaliser une analyse fourragère et de faire ressortir l'impact du facteur suscité. Dans ce contexte, nous avons comparé l'offre fourragère de cinq espèces appartenant aux familles suivantes: Poaceae, Fabaceae, Plantaginaceae et Papaveraceae, cette comparaison a aboutit à un déficit de 18630,27 UFL, c'est-à-dire un manque de $22975,07 \mathrm{~kg}$ de matière sèche pour les six stations. L'analyse factorielle des correspondances (AFC) nous a permis d'identifier deux groupes de stations: le premier groupe (G1) est représenté par les stations S2, S5, et S6 dont l'impact du pâturage est faible parfois moyen. Le deuxième (G2) représente les stations les plus pâturées S1, S3 et S4, reflétant ainsi une banalisation du cortège floristique de ces dernières.
\end{abstract}

Mots clés: Surpâturage, Phytodiversité, Mont de Tessala, plantes broutées, analyse fourragère, factorielle des correspondances.

\begin{abstract}
Influence of grazing on phytodiversity and variation in the chemical composition of five grazed plants in the Mount of Tessala (north-western Algeria)

The aim of our work is to assess the impact of grazing on the plant biodiversity of the Tessala mountains. For this, six (06) stations located on the north and south slopes of the study area were sampled. At each station, a floristic study and soil analyzes are carried out in order to identify the main factors involved in the distribution of vegetation. In addition, field surveys were carried out in order to carry out a forage analysis and highlight the impact of the factor raised. In this context, we compared the forage supply of five species belonging to the following families: Poaceae, Fabaceae, Plantaginaceae and Papaveraceae, this comparison resulted in a deficit of 18630,27 UFL, that is to say a lack of $22975,07 \mathrm{~kg}$ dry matter for the six stations. The factorial correspondence analysis (FCA) has enabled us to identify two groups of stations: the first group (G1) is represented by stations S2, S5, and S6, whose impact from grazing is sometimes low. The second (G2) represents the most grazed stations S1, S3 and S4, thus reflecting a trivialization of the floristic procession of the latter.
\end{abstract}

Key words: overgrazing, phytodiversity, Tessala mountains, grazed plants, forage analysis, Factorial correspondence anaysis.

\section{Introduction}

La surexploitation de la forêt et les pratiques agro-pastorales intensives en régions méditerranéennes ont conduit jusqu'au siècle dernier à une forte dégradation des formations forestières (Emberger, 1971). Ainsi, le patrimoine forestier de l'Algérie occi- dentale et particulièrement celui des monts de Tessala (nord ouest algérien), connaît depuis des décennies une continuelle régression due aux pratiques irrationnelles de l'homme (déboisements, surpâturages, incendies etc.) (Cherifi et al., 2017), et au changement péjoratif du climat, caractérisé par une sécheresse estivale prolongée et une irrégularité 
des pluies (Quézel, 2000). Une telle évolution a provoqué la substitution d'une végétation mésophytique d'origine, par une végétation xérophytique (Gaouar, 1980; Bouazza et al., 2004; Cherifi et al., 2011).

Une de ces causes de dégradation est l'élevage pastoral qui se considère l'une des bases de l'organisation économique et sociale des populations dans les zones forestières. Cette activité peut entrainer des dégâts néfastes sur la dynamique, le recouvrement et la richesse spécifique des parcours forestiers et steppique (Benabdeli, 2000).

Le massif montagneux de la zone d'étude présente un réel intérêt de par ses composantes tant géographiques qu'écologiques. Son rôle environnemental et socio-économique mérite d'être signaler et étudier. Ce massif abrite une faune et une flore d'un intérêt particulier mais qui restent soumises continuellement à des pressions humaines croissantes et incontrôlées. Ces dernières consti-tuent une menace directe pour la préservation et le renouvellement des ressources biologiques ainsi que pour l'équilibre écologique de la zone (Cherifi et al., 2011; Cherifi, 2013). II convient enfin de souligner que cet écosystème fragilisé se place dans un contexte environnemental extrêmement important de préservation de la biodiversité végétale, sachant qu'il n'a fait l'objet que de recherches limitées et que la connaissance de sa structure et de son fonctionnement n'en est qu'à ses débuts (Cherifi et al., 2011). La majorité des investigations effectuées sur l'écosystème forestier du mont de Tessala portent sur l'inventaire des plantes (Baraka, 2008; Mokaddem, 2012; Ouici, et al., 2015; Hakemi et al., 2018) et sur la valorisation biochimique de certains taxons à caractère médicinal (Bouterfas, 2011; Bouzidi et al., 2012). Les travaux sur l'évaluation de l'impact de l'action anthropozoogène sur la couverture végétale du massif forestier du mont de Tessala sont limités (Cherifi et al., 2011, 2014, 2017).

Ces considérations nous ont conduits à mettre en place une étude de l'influence du pâturage sur la diversité floristique et la phytomasse herbacée de cinq espèces appartenant aux familles suivantes: Poaceae, Fabaceae, Plantaginaceae et Papaveraceae.

\section{Matériel et méthodes}

\section{Site d'étude et localisation des stations d'échantillonnage}

Le mont de Tessala est un massif montagneux situé au nord de la wilaya de Sidi Bel Abbés. II est limité au Nord par la plaine de la Mleta et la Sebkha d'Oran, à l'Est par les monts des Béni Chougrane, à l'Ouest par les monts de Sebaa Chioukh et au Sud par la plaine de Sidi Bel Abbés. Cette chaine montagneuse de l'Atlas tellien culmine à $1061 \mathrm{~m}$ d'altitude (Cherifi et al., 2017) (Figure 1).
Le mont de Tessala fait partie du Tell oranais central. Sa constitution géologique est marquée par des formations de nappes de charriage du complexe Crétacé-Oligocène et des formations à dominance calcaire peu résistantes à l'érosion. Des affleurements de grés calcaires apparaissent dans des endroits des semelles de gypse du Trias (Kiekken, 1962).

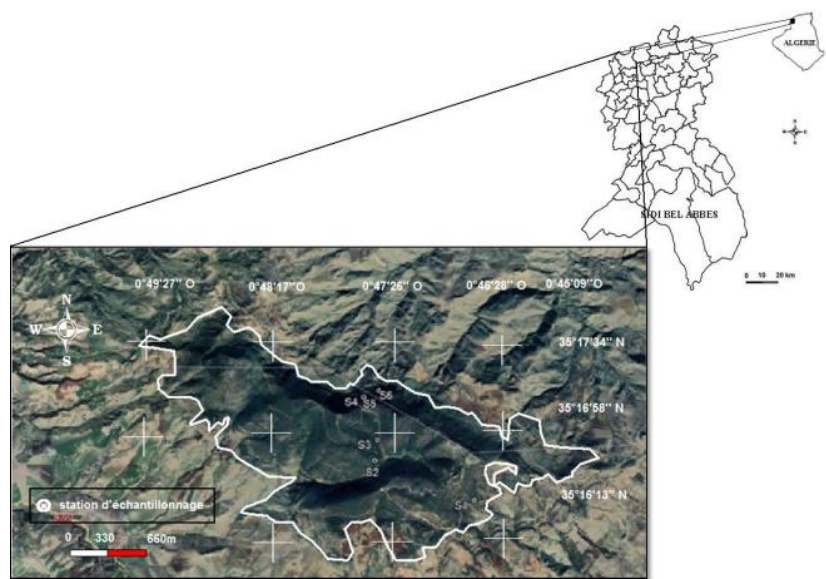

Figure 1. Situation géographique du mont de Tessala et localisation des stations d'échantillonnages.

Figure 1. Geographical location of Mount Tessala and location of the sampling stations.

Le climat est de type méditerranéen semi-aride à hiver frais, avec une pluviométrie annuelle moyenne de $335-400 \mathrm{~mm}$ et une température annuelle moyenne de $8-26^{\circ} \mathrm{C}, 2^{\circ} \mathrm{C}$ pour décembre et $30^{\circ} \mathrm{C}$ pour Août (Cherifi et al., 2017).

\section{Méthodologie}

Le travail sur terrain consiste à choisir des emplacements (stations) aussi typiques que possible. Ce choix est basé sur l'intensité du pâturage, la physionomie de la végétation et l'exposition. De ce fait six (06) stations situées sur les versants nord et sud de la zone d'étude ont été choisies (Figure 1, Tableau 1).

Au niveau de chaque station et pendant la période propice de végétation optimale (mars- début juin, 2016), ont été réalisés trois (03) relevés phytoécologiques, soit au total dix white (18) relevés suivant la méthode sigmatiste (Braun-Blanquet, 1951). La superficie de chaque relevé est de $100 \mathrm{~m}^{2}$, une surface floristiquement homogène et représentative permettant la collecte d'informations fiables (Gounot, 1969). La valeur de l'aire minimale peut être appréciée assez facilement; elle est sensiblement constante pour les divers relevés d'un groupement déterminé, mais varie beaucoup d'un groupement à l'autre (Ozenda, 1982). L'inventaire floristique est basé sur les strates arborescente, arbustive, buissonnante et herbacée. Chaque espèce recensée a été affectée d'un coefficient d'abondance-dominance et de sociabilité (Cherifi et al., 2017). L'identification des espèces est complétée 
selon la flore de Quézel et Santa (1962), Quézel et Santa (1963), APG III (2009) \& Tela Botanica (2015). Les caractères stationnels, à savoir l'altitude, la pente, l'exposition, la nature du substrat et le taux de recouvrement moyen sont notés. Au terme des 18 relevés, une liste regroupant toutes les espèces recensées est établie.

Tableau 1. Description et localisation des stations d'échantillonnage.

Table 1. Description and location of the sampling stations.

\begin{tabular}{|c|c|c|c|c|}
\hline Stations & Latitude Nord & Longitude Ouest & Intensité de pâturage & Exposition \\
\hline $\begin{array}{l}\text { S1 (garrigue à base de calicotome et } \\
\text { palmier nain) }\end{array}$ & $35^{\circ} 16^{\prime} 174^{\prime \prime} \mathrm{N}$ & $00^{\circ} 46^{\prime} 253^{\prime \prime} \mathrm{W}$ & intense & Versant sud \\
\hline S2 (forêt claire de pin d'Alep) & $35^{\circ} 16^{\prime} 335^{\prime \prime} \mathrm{N}$ & $00^{\circ} 47^{\prime} 176^{\prime \prime} \mathrm{W}$ & moyen & Versant sud \\
\hline $\begin{array}{l}\text { S3 (garrigue à base de calicotome et } \\
\text { palmier nain) }\end{array}$ & $35^{\circ} 16^{\prime} 425^{\prime \prime} \mathrm{N}$ & $00^{\circ} 46^{\prime} 885^{\prime \prime} \mathrm{W}$ & intense & Versant sud \\
\hline $\begin{array}{l}\text { S4 (garrigue à base de calicotome et } \\
\text { diss) }\end{array}$ & $35^{\circ} 17^{\prime} 021^{\prime \prime} \mathrm{N}$ & $00^{\circ} 47^{\prime} 024^{\prime \prime} \mathrm{W}$ & intense & Versant nord \\
\hline S5 (matorral à base de chêne vert) & $35^{\circ} 16^{\prime} 984^{\prime \prime} \mathrm{N}$ & $00^{\circ} 46^{\prime} 763^{\prime \prime} \mathrm{W}$ & faible & Versant nord \\
\hline S6 (matorral à base de chêne vert) & $35^{\circ} 17^{\prime} 069^{\prime \prime} \mathrm{N}$ & $00^{\circ} 47^{\prime} 768^{\prime \prime} \mathrm{W}$ & faible & Versant nord \\
\hline
\end{tabular}

Des analyses concernent la texture, l'humidité, la conductivité électrique, le $\mathrm{pH}$, le taux de calcaire et le taux de matière organique ont été effectuées sur chaque station. Les valeurs relatives à ces différents paramètres physico-chimiques correspondent à des valeurs moyennes par station.

Sur la partie du végétal récolté, des essais pour estimer l'offre fourragère des espèces choisies ont été effectués pour l'ensemble des stations échantillonnées. Cinq (05) espèces de type fourrager appartenant à différentes familles: deux espèces de la famille des Poaceae (Aegilops triuncialis L. et Agropyrum repens L.) une Plantaginaceae (Plantago lagopus L.), une Papaveraceae (Papaver rhoeas L.) et l'autre Fabaceae (Trifolium stellatum L.) ont été sélectionnés en fonction du niveau d'appétibilité par les animaux.

Le prélèvement des espèces est effectué avant le début du pâturage pour bien rationner l'alimentation de cheptel. Les échantillons de chacune des plantes sont mélangés dans un sachet accompagnés d'une fiche des renseignements (nom, groupe, poids, date, station) pour le transport au laboratoire.

Après le stockage, chaque échantillon de l'espèce est broyé à l'aide d'un broyeur suivi par un tamisage. Le poids de la prise est de $5 \mathrm{~g}$ pour chaque échantillon à analyser. Les analyses effectuées concernent l'humidité, la matière sèche (MS), la matière organique $(\mathrm{MO})$, la matière minérale $(\mathrm{MM})$ et dosage de la cellulose brute (CB) afin d'estimer les besoins fourragers du cheptel au niveau de la zone d'étude.

\section{Traitement des données}

Les méthodes multi variées comme l'analyse multidimensionnelle sont actuellement considérées comme les mesures les plus sensibles pour ce qui est de la détection des changements affectant la structure de la communauté, notamment si on compare aux mesures traditionnelles de la biodiversité (Clarke \& Warwick, 2001).
Le traitement des données floristiques, pédologiques et analyses fourragères a été réalisé par l'analyse factorielle des correspondances (AFC) et la classification hiérarchique ascendante ( $\mathrm{CHA})$. Cette approche est bien adaptée aux études phytosociologiques et permet de traiter les variables floristiques et pédologiques (Djebaili, 1984). C'est un outil qui discrimine, de manière objective, les entités particulières ou de comparer les stations deux à deux (Bonin \& Tatoni, 1990). La classification hiérarchique ascendante permet de mieux individualiser les limites entre les différents groupements (Benzécri, 1984; Cherifi et al., 2014, 2017). La matrice de l'AFC croise l'ensemble des variables édaphiques (07 paramètres), les types biolologiques, le taux de recouvrement de la végétation, l'altitude et le facteur pâturage pour la mise en évidence des principaux gradients écologiques pour les six stations échantillonnées.

\section{Résultats et discussion}

\section{Composition floristique}

L'inventaire floristique réalisé au niveau des six stations, soit 18 relevés au total, a permis de recenser 92 espèces rattachées à 78 genres (Tableau 2). Le nombre d'espèces par relevé varie entre 72 (relevé R3) et 29 (relevé R18), soit une moyenne de 70 espèces (station $\mathrm{S} 1$ ) à 42 espèces (station 6). Selon la classification de Daget \& Poissonet (1997), cette diversité floristique des six stations étudiées peut être qualifiée de moyenne.

\section{Spectre biologique}

La classification des espèces recensées selon leur type biologique montre que les thérophytes présentent un taux très élevé de $37 \%$, suivies par les hémicryptophytes avec $25 \%$, les chaméphytes avec 
Tableau 2. Relevés floristiques effectués sur terrain. Coefficient d'abondance-dominance et de sociabilité séparés par un point. F: indice de présence ou fréquence d'apparition d'une espèce dans un groupement en pourcentage, I: $20 \%$; II: 20-40; III: 40-60; IV: 60-80\%; V:> 80.

Table 2. Floristic surveys carried out in the field. Coefficient of abundance-dominance and sociability separated by a point. F: index of presence or frequency of occurrence of a species in a group expressed as percentage, I: 20\%; II: 2040; III: 40-60; IV: 60-80\%; V:> 80.

\begin{tabular}{|c|c|c|c|c|c|c|c|c|c|c|c|c|c|c|c|c|c|c|c|c|}
\hline Localisation & S1 & & & $\mathrm{S} 2$ & & & S3 & & & S4 & & & S5 & & & S6 & & & $F(\%)$ & \\
\hline Altitude (m) & 806 & & & 927 & & & 877 & & & 868 & & & 987 & & & 909 & & & & \\
\hline Pente (\%) & 16 & & & 16 & & & 16 & & & 18 & & & 18 & & & 16 & & & & \\
\hline Exposition & Sud & & & Sud & & & Sud & & & Nord & & & Nord & & & Nord & & & & \\
\hline $\begin{array}{l}\text { Taux de } \\
\text { recouvrement } \\
\text { moyen (\%) }\end{array}$ & 20 & & & 30 & & & 20 & & & 20 & & & 35 & & & 35 & & & & \\
\hline Relevés & $\mathrm{R} 1$ & $\mathrm{R} 2$ & $\mathrm{R} 3$ & $\mathrm{R} 4$ & R5 & $\mathrm{R} 6$ & $\mathrm{R} 7$ & $\mathrm{R} 8$ & $\mathrm{R} 9$ & $\mathrm{R} 10$ & $\mathrm{R} 11$ & $\mathrm{R} 12$ & $\mathrm{R} 13$ & $\mathrm{R} 14$ & $\mathrm{R} 15$ & $\mathrm{R} 16$ & $\mathrm{R} 17$ & $\mathrm{R} 18$ & & \\
\hline \multicolumn{21}{|c|}{ Strate arborescente } \\
\hline Arbutus unedo $\mathrm{L}$. & . & . & . & . & . & . & . & . & . & . & . & . & . & . & . & . & 1.1 & 1.1 & 11.11 & I \\
\hline Viburnum tinus $\mathrm{L}$. & . & . & . & . & . & . & & . & . & . & . & . & . & 1.1 & 1.1 & . & 1.1 & 1.1 & २2.२2 & II \\
\hline $\begin{array}{l}\text { Crataegus } \\
\text { monogyna Jacq. }\end{array}$ & . & . & . & . & +.2 & . & +.2 & . & & +.2 & . & . & 1.1 & 1.1 & 1.1 & 2.2 & 1.1 & 2.2 & 50 & III \\
\hline $\begin{array}{l}\text { Olea europaea } \\
\text { Var. Oleaster Dc. }\end{array}$ & +.1 & . & +.2 & . & 1.1 & +.2 & 1.1 & +.1 & 1.1 & . & . & . & . & . & . & . & . & . & 38.88 & II \\
\hline $\begin{array}{l}\text { Pinus halepensis } \\
\text { Mill. }\end{array}$ & . & . & . & 3.3 & 2.2 & 3.3 & +.2 & +.2 & . & . & . & . & . & . & . & . & . & . & 27.77 & II \\
\hline Quercus ilex L. & . & . & . & 2.2 & 1.1 & 1.1 & 1.1 & 3.3 & 1.1 & 1.1 &.++ & 1.1 & 3.3 & 3.3 & 3.3 & 4.4 & 3.3 & 3.3 & 83.33 & V \\
\hline $\begin{array}{l}\text { Quercus } \\
\text { coccifera L. }\end{array}$ & . & . & +.2 & +.2 & 1.1 & +.2 & . & . & . & 2.2 & . & . & 2.2 & 2.2 & 2.2 & 2.2 & 2.2 & 3.3 & 61.11 & IV \\
\hline \multicolumn{21}{|c|}{ Strate arbustive } \\
\hline $\begin{array}{l}\text { Rosa } \\
\text { sempervirens L. }\end{array}$ & . & . & . & . & +.2 & . & 1.1 & 2.2 & 1.1 & . & 1.1 & 2.2 & 1.1 & 1.1 & 1.1 & 1.1 & 1.1 & 1.1 & 66.66 & IV \\
\hline $\begin{array}{l}\text { Pistacia } \\
\text { lentiscus L. }\end{array}$ & . & . & +.2 & 1.1 & 1.1 & 2.2 & 2.2 & 2.2 & 2.2 & . & . & . & 2.2 & 3.3 & 2.2 & 2.2 & 2.2 & 2.2 & 72.22 & IV \\
\hline $\begin{array}{l}\text { Pistacia } \\
\text { terebinthus L. }\end{array}$ & . & . & . & . & +.2 & 1.1 & 2.2 & 1.1 & 1.1 & . & . & . & 1.1 & 1.1 & 1.1 & 1.1 & 1.1 & 1.1 & 61.11 & IV \\
\hline $\begin{array}{l}\text { Pistacia x } \\
\text { saportae Burnat. }\end{array}$ & . & . & . & . & 1.1 & . & . & . & . & . & . & . & . & . & . & . & . & . & 5.55 & I \\
\hline \multicolumn{21}{|c|}{ Strate buissonnante et herbacée } \\
\hline $\begin{array}{l}\text { Aegilops } \\
\text { triuncialis L. }\end{array}$ & 2.2 & 2.2 & 1.1 & . & 1.1 & 2.2 & 2.2 & 1.1 & 1.1 & 2.2 & 2.2 & 2.2 & 2.2 & 1.1 & 2.2 & 2.2 & 1.1 & 1.1 & 94.44 & $\mathrm{~V}$ \\
\hline $\begin{array}{l}\text { Agropyrum } \\
\text { repens L. }\end{array}$ & 2.2 & 2.2 & 1.1 & 2.2 & 2.2 & 1.1 & 2.2 & 1.1 & 1.1 & 2.2 & 2.2 & 2.2 & 2.2 & 1.1 & 1.1 & 2.2 & . & 1.1 & 94.44 & $\mathrm{~V}$ \\
\hline $\begin{array}{l}\text { Ajuga iva (L.) } \\
\text { Schreb. }\end{array}$ & 2.2 & 1.1 & 1.1 & 1.1 & . & . & 1.1 & 1.1 & 1.1 & . & . & . & . & . & . & . & . & . & 38.88 & II \\
\hline $\begin{array}{l}\text { Ammi visnaga } \\
\text { (L.) Lam. }\end{array}$ & 2.2 & 1.1 & 1.1 & . & . & . & . & . & . & 1.1 & 1.1 & 1.1 & . & . & . & . & . & . & 33.33 & II \\
\hline $\begin{array}{l}\text { Ampelodesmos } \\
\text { mauritanicus } \\
\text { (Poir.) }\end{array}$ & . & +.2 & +.2 & 1.1 & 1.1 & . & . & . & 2.2 & 3.3 & 3.3 & 3.3 & 1.1 & 2.2 & 1.1 & 1.1 & . & 1.1 & 72.22 & IV \\
\hline $\begin{array}{l}\text { Anagallis } \\
\text { arvensis } \mathrm{L} .\end{array}$ & 1.1 & 1.1 & . & . & 1.1 & +.2 & 1.1 & 1.1 & 1.1 & 2.2 & 2.2 & 2.2 & 1.1 & . & +.2 & +.2 & . & +.2 & 77.77 & IV \\
\hline Anagallis monelli $\mathrm{L}$. & 1.1 & 1.1 & 1.1 & . & 1.1 & 1.1 & 1.1 & 1.1 & 1.1 & 2.2 & 1.1 & 2.2 & 1.1 & . & 1.1 & 2.2 & 2.2 & 1.1 & 88.88 & V \\
\hline $\begin{array}{l}\text { Anchusa azurea } \\
\text { Mill. }\end{array}$ & 1.1 & . & +.1 & . & +.1 & . & 1.1 & 1.1 & +.2 & . & . & . & +.2 & . & . & . & +.2 & & 44.44 & III \\
\hline $\begin{array}{l}\text { Anacyclus } \\
\text { pyrethrum (L.) } \\
\text { Lag. }\end{array}$ & +.2 & 1.1 & 1.1 & +.2 & . & . & 1.1 & +.2 & 1.1 & . & . & . & 1.1 & . & . & . & 1.1 & . & 50 & III \\
\hline
\end{tabular}




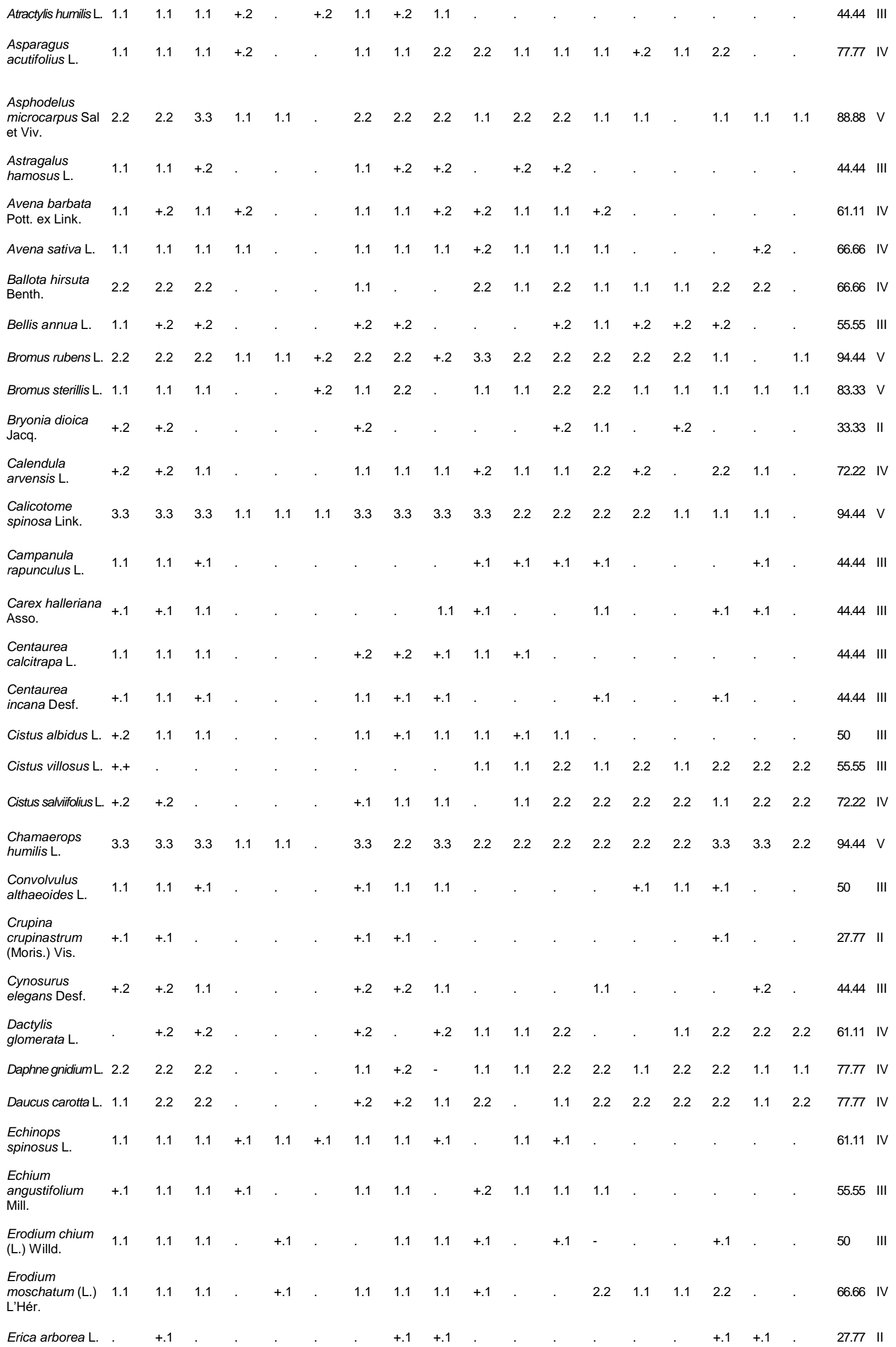


Eryngium

Euphorbia

chamaesyce $\mathrm{L}$.

27.77 II

Foeniculum

vulgare Mill.

$\begin{array}{lll}1.1 & 1.1 & 1.1\end{array}$

$1.1 \quad 1.1 \quad 1.1 \quad 1$.

1.1

Galium aparine L. .

$1.1 \quad 1.1 \quad 1.1$

Helianthemun

polyanthum

(Desf.) Pers.

Hordeum

bulbosum $\mathrm{L}$.

$\begin{array}{lll}1.1 & 2.2 & 2.2\end{array}$

$\begin{array}{lll}1.1 & 2.2 & 2.2\end{array}$

$1.1 \quad 1.1$

72.22 IV

Hordeum

murinum $\mathrm{L}$.

$2.2 \quad 2.2 \quad 2.2$

$1 \quad 1.12$

2.2

$2 \quad 1.1$

$\begin{array}{llllll}1.1 & 1.1 & 1.1 & 1.1 & 1.1 & 1.1\end{array}$

1.1

33.33 II

$\begin{array}{llll}\text { Iris spuria L. } \quad 2.2 & 2.2 & 2.2\end{array}$

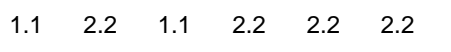

$\begin{array}{llll}1.1 & 1.1 & 2.2 & 1.1\end{array}$

$83.33 \mathrm{~V}$

Lagurus ovatus L. $2.2 \quad \begin{array}{lllll}1.1 & 2.2 & 1.1 & 1.1\end{array}$.

$2.2 \quad 2.2 \quad 1.1$

. 1.1

44.44 III

Linum strictum L. $1.1 \quad 1.1 \quad 1.1$

$2.2 \quad 1.1 \quad 2.2$

$2.2 \quad 2.2 \quad 1.1$

2.2

72.22 IV

Lobularia

maritime (L.) $\quad 2.2 \quad 2.2 \quad 2.2$

$1.1 \quad 1.1 \quad 1.1$

$1.1 \quad 1.1$

$50 \quad$ III

Desv.

$\begin{array}{llllll}2.2 & 2.2 & 2.2 & 2.2 & 1.1 & 2.2\end{array}$

55.55 III

Lonicera implexa

Aiton.

55.55 III

Lotus

2.2

$\begin{array}{lllllllll}1.1 & 1.1 & 2.2 . & 2.2 & 2.2 & 2.2 & 2.2 & 2.2 & 1.1\end{array}$

$\begin{array}{llll}1.1 & 2.2 & 2.2 & 1.1\end{array}$

$88.88 \mathrm{~V}$

corniculatus $\mathrm{L}$.

1.1

$+.2$

16.66 I

Marrubium

vulgare $\mathrm{L}$.

$2.2 \quad 2.2 \quad 2.2$

$1.1 \quad 1.1$

$1.1 \quad 1.1 \quad$.

$38.88 \quad$ II

Medicago $2.2 \quad 1.1 \quad 1.1$

1.1 .1 .1

2.2

33.33 II

Muscari

comosum (L.) $\quad 1.1 \quad 1.1 \quad 1.1$

Mill.

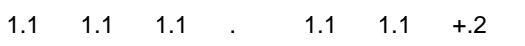

$+.2$

55.55 III

Paronychia

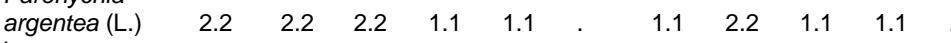

Lam.

Papaver

hybridum L.

Papaver rhoeas L. $2.2 \quad 2.2 \quad 1.1$

$1.1+1.1+1$

38.88 II

Phillyrea

$\begin{array}{llllll}1.1 & 2.2 & 2.2 & 1.1 & 2.2 & 2.2\end{array}$

61.11 IV

angustifolia $\mathrm{L}$.

1.11 .1

$1.1 \quad 1.1$

22.22 II

$\begin{array}{llll}\begin{array}{l}\text { Plantago } \\ \text { albicans L. }\end{array} & 2.2 & 2.2 & 3.3\end{array}$

$1.1 \quad 1.1$

$\begin{array}{lllll}1.1 & 1.1 & 2.2 & 2.2 & 1.1\end{array}$

66.66 IV

Plantago

lagopus $\mathrm{L}$.

$2.2 \quad 2.2 \quad 3.3$

$\begin{array}{llll}1.1 & 1.1 & 1.1 & 2.2\end{array}$

$\begin{array}{lllllll}2.2 & 2.2 & 2.2 & 1.1 & 1.1 & 2.2 & 1.1\end{array}$

77.77 IV

Pulicaria odora

(L.) Rchb.

. $1.1+.1$

16.66 ।

$\begin{array}{lllll}\text { Reseda alba L. } & 2.2 & 2.2 & 2.2 & 1.1\end{array}$.

$2.2 \quad 1.1 \quad 2.2$

$2.2 \quad 2.2 \quad 1.1$

55.55 III

Rubus ulmifolius

Schott.

Ruta

chalepensis

$2.2 \quad 3.3 \quad 22$

$1.1 \quad 1.1$

$1.1 \quad 1.1$

$1.1+$

$2.2 \quad 1.1 \quad 2.21 .1$

Ruta montana L. $\begin{array}{lll}3.3 & 2.2 & 2.2\end{array}$

$\begin{array}{llllll}1.1 & 1.1 & 2.2 & 1.1 & 2.2 & 1.1\end{array}$

Salvia argentea L.

1.1

$2.2 \quad 1.1$

Scabiosa stellata L. $2.2 \quad 1.1 \quad 2.2$

$\begin{array}{llll}1.1 & 1.1 & 2.2 & 1.1\end{array}$.

$1.1 \quad 1.1 \quad 1.1$

2.2

Scolymus

hispanicus L.

$\begin{array}{lllll}1.1 & 2.2 & 1.1 & 1.1 & 1.1\end{array}$

2.2

2.2

61.11 IV

Silybium

$\begin{array}{llll}\text { marianum Poir. } & 2.2 & 1.1 & 2.2\end{array}$

1.1 


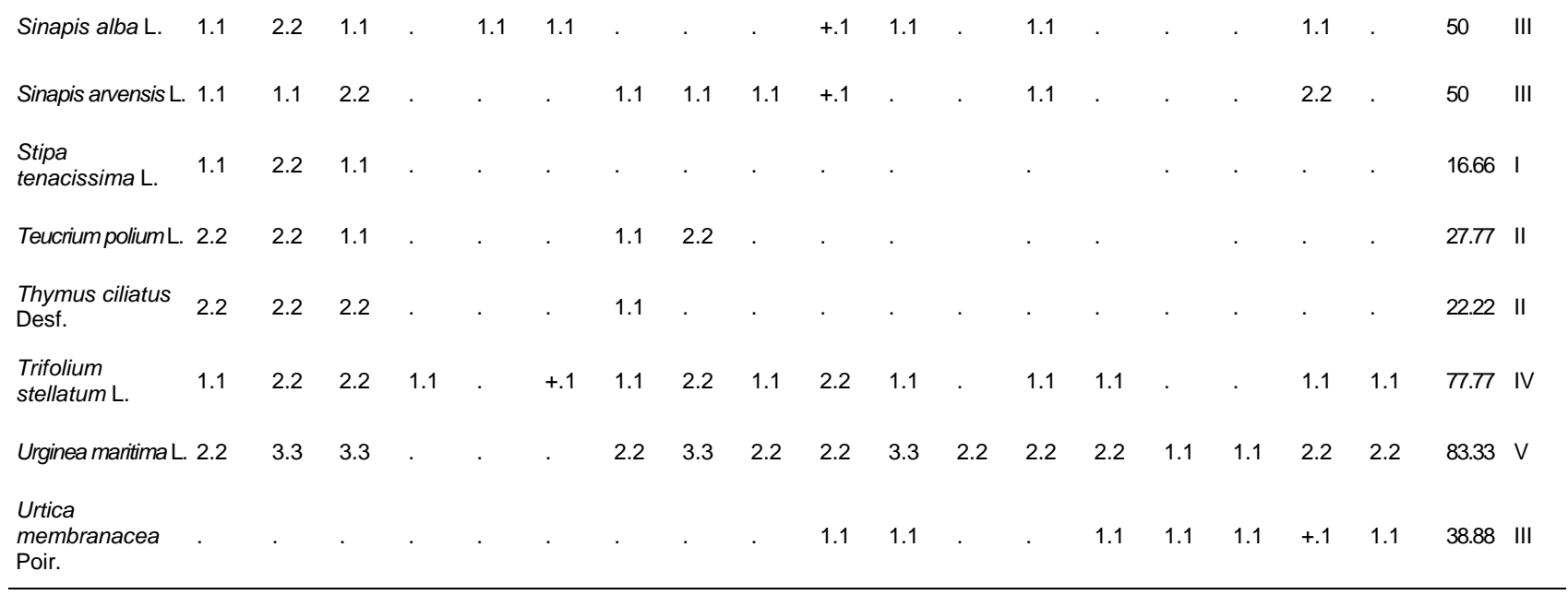

$14 \%$, les nanophanérophytes avec 11\%, les géophytes avec $8 \%$ et les phanérophytes avec $5 \%$.

La végétation étudiée est caractérisée actuellement par le type: $\mathrm{Th}>\mathrm{He}>\mathrm{Ch}>\mathrm{Na} . \mathrm{Ph}>\mathrm{Ge}$ $>\mathrm{Ph}$. La dominance des thérophytes dans l'ensemble des stations étudiées est lié aux pâturages fréquents et surtout à des cultures riveraines. La prédominance des thérophytes dans l'ensemble des stations étudiées reflète l'état dégradé de l'écosystème forestier du massif de Tessala (Cherifi et al., 2011). Les périodes de sécheresse prolongée dans cette zone durant les deux dernières décennies seraient à l'origine de la dominance de ce type biologique (Quézel 2000). En effet, la thérophytisation est qualifiée comme une forme de résistance à la sécheresse et représente un stade de dégradation ultime (Kadi Hanifi, 2003; Barbero et al., 1990; Cherifi et al., 2011, 2017). Les variations du spectre biologique des espèces recensées s'explique par l'étagement altitudinal, les fluctuations locales des paramètres bioclimatiques ainsi que par les pressions multiples exercées par l'homme et son bétail (Sauvage, 1961).

\section{Caractérisation des familles}

Les 92 espèces recensées se rattachent à 36 familles botaniques (Figure 2). La famille la mieux représentée est celle des Poaceae (13 espèces, soit $14,28 \%$ ) suivies par les Asteraceae (12 taxons, soit $13,18 \%$ ), ensuite viennent les Fabaceae et les Lamiaceae (5 espèces, soit $5,49 \%$ ), puis les Apiaceae et les Cistaceae (4 espèces, soit 4,39\%), les Liliaceae, les Rosaceae, les Brassicaceae, les Anacardiaceae (3 espèces, soit 3,29\%), les Caprifoliaceae, les Primulaceae, les Boraginaceae, les Ericaceae, les Geraniaceae, les Oleaceae, les Papaveraceae, les Fagaceae, les Rutaceae et les Plantaginaceae (2 espèces, soit $2,19 \%$ ). Les 17 familles restantes sont chacune représentées par une espèce soit $1,09 \%$.

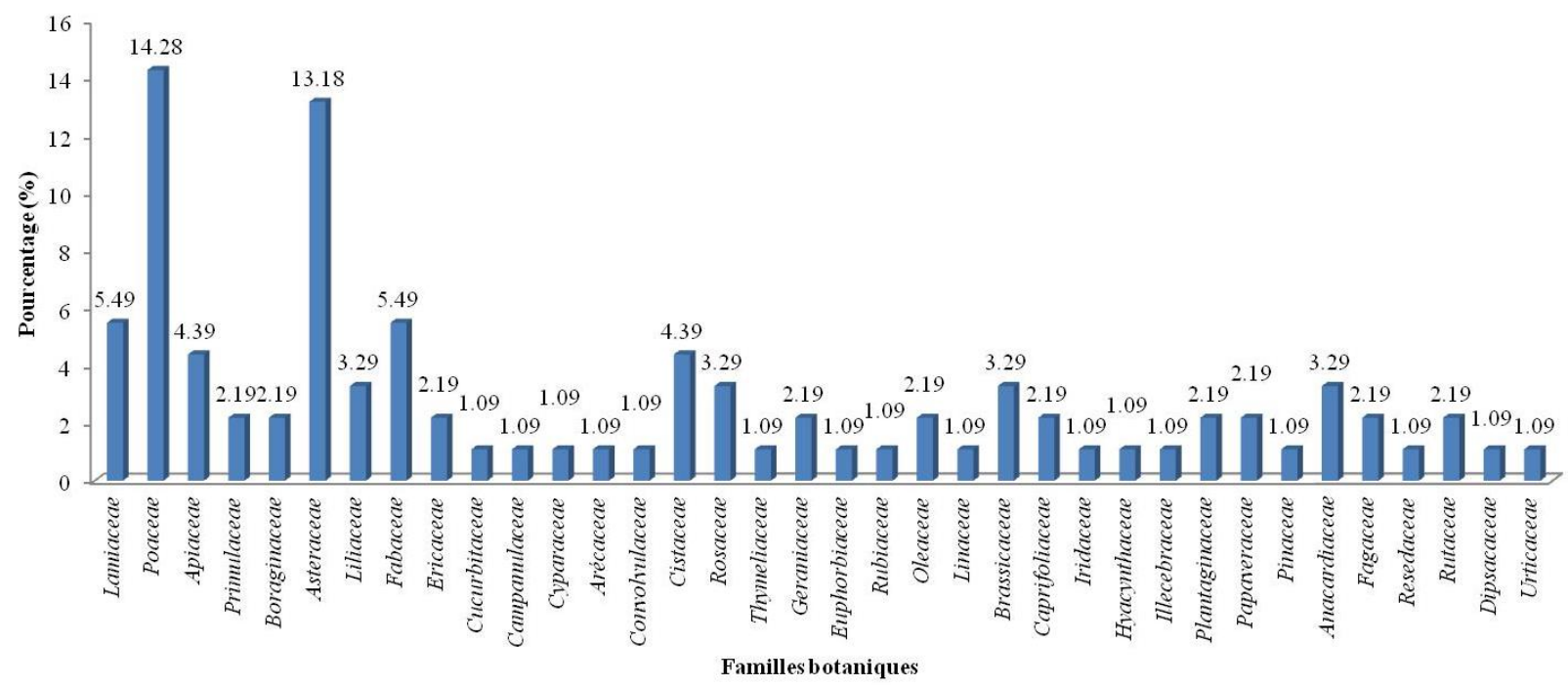

Figure 2. Taux de différentes familles botaniques des espèces recensées.

Figure 2. Rates of different botanical families of the species recorded. 


\section{Origine biogéographique}

La caractérisation phytogéographique montre que la recensée est très diversifiée et comprend plusieurs groupes phytochorologiques (Figure 3). Les espèces recensées se caractérisent par la présence de 2 espèces endémique nord africaine, soit 2,19\%. L'ensemble méditerranéen reste le plus représentatif avec 42 espèces, soit 46,15\%. Successivement, se succèdent les éléments ouestméditerranéens, euro-méditerranéens et paléotempérés (5 espèces, soit $5,49 \%$ ), méditerranéenneirano-touranienne, ibéro-mauritaniques, eurasiatiques, cosmopolites (4 espèces, soit 4,39\%), macaronésienne-méditerranéennes (3 espèces, soit $3,29 \%)$. Les autres types biogéographiques sont pratiquement représentés uniquement par une à deux espèces.

\section{Analyse du sol}

Les valeurs moyennes relatives aux différents paramètres physico-chimiques, caractéristiques de l'horizon superficiel du sol, sont représentées dans le Tableau 3.

Les sables dominent dans la majorité des stations étudiées avec des proportions variantes de $19 \%$ à $50,58 \%$. Les taux de sable déterminent ainsi une texture sablo-argileuse notamment pour les stations S3, S4 et S6 avec respectivement 30,40\%, 50,58\% et $44,30 \%$. Le taux des limons reste moins importants comparativement aux argiles. On enregistre une augmentation de l'argile en S1, S2 et S5 avec respectivement $33,26 \%, 25,70 \%$ et $21,91 \%$ offrant ainsi une texture argilo-sableuse. Le taux d'humidité varie d'une station à une autre selon l'exposition. Elle est plus importante dans les stations du versant nord $(H>10,80 \%)$. Dans les stations du versant sud, elle ne dépasse guère point $8,31 \%$. L'exposition prolongée à l'éclairement et aux vents chauds du versant sud favorisent l'évaporation de l'humidité du sol, en plus le versant nord est exposé aux vents humides venant de la mer ce qui favorisent l'augmentation de la charge en eau dans le sol (Cherifi et al., 2011). Le pH reste légèrement alcalin, compris entre 7,23 et 7,60 avec une différence très peu significative entre les différents échantillons analysés. La conductivité électrique révèle des sols non salés pour la plupart des échantillons. La teneur des sols en matière organique varie de $3,50 \%$ à $4,42 \%$. Une forte teneur est enregistrée au niveau des stations S2, S5 et S6 avec respectivement $4 \%, 4,05 \%$ et 4,42 . Pour les autres stations S1, S3 et S4, le taux varie de 3,50\% à $3,75 \%$ traduisant des sols à teneurs moyennes. Le taux de calcaire total oscille entre 1,14 à $24,16 \%$. Les sols des stations S5 et S6 sont peu calcaire avec un taux variant de $1,14 \%$ à $1,66 \%$. La station S4 est caractérisée par un sol modérément calcaire avec un taux de $2,77 \%$. Un sol fortement calcaire est enregistré au niveau des stations S1, S2 et S3 dont le taux varie de $20,16 \%$ à $24,16 \%$.

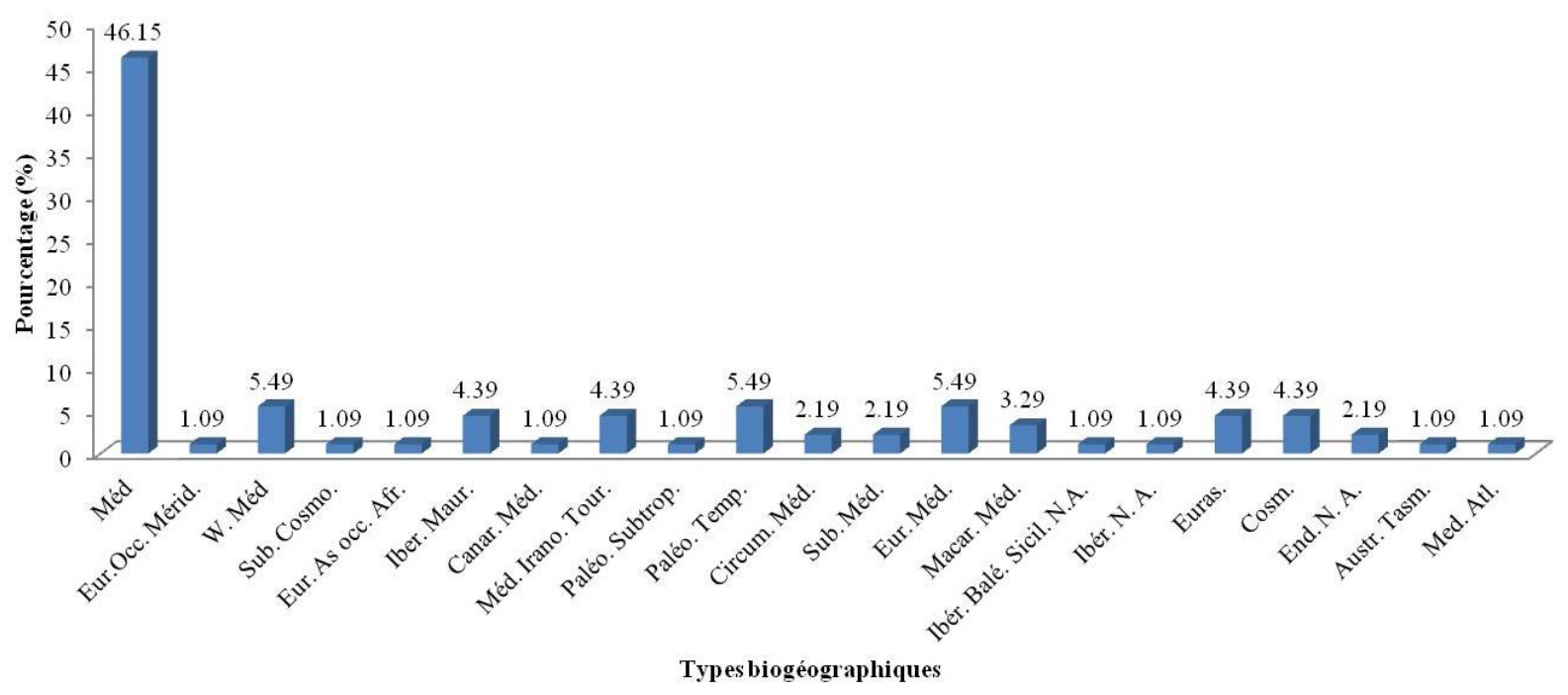

Figure 3. Taux de différents éléments biogéographiques des espèces recensées. Légende: Med: Méditerranéenne; WMed: Ouest-Méditerranéenne; Euras: Eurasiatique; Eur-Med: Euro-Méditerranéenne; Cosm: Cosmopolite; Ibero-Maur.: Ibéro-Mauritanique; Circum. Méd.: Circum- Méditerranéenne; Macar-Med: Macaronésienne Méditerranéenne; Macar-Med.: Macaronésienne Méditerranéenne; Med.-Irano-Tour: Méditerranéenne-Irano-Touranienne; Paleo-Subtrop: Paléo-SubTropicale; Paleo-Temp: Paléo-Tempérée; Sub.Cosmop: Sub-Cosmopolite; Sub-méd.: Sub-Méditerranéenne; Méd. Atl.: Méditerranéen Atlantique; End. N. A.: Endémiques nord-africaines; Aust. Tasm.: Australien -tasanie; Ibér. N. A.: IbéroNord-Afrique; Ibéro. Balé. Sicil.: Ibéro-Balé-Sicile; Eur. Méd: Eurasiatique-Méditerranéen; Canar. Méd: CanariensMéditerranéennes; Eur. Occ. Mérid.: Européen. Occidentale-Méridionale.

Figure 3. Rate of different biogeographical elements of the species listed. 


\section{Analyses fourragères}

Teneur en matière sèche, humidité, matière organique et matière minérale

Les teneurs en matière sèche, humidité, matière organique et matière minérale des espèces étudiés sont présentés ci-après (Figure 4).

La teneur en matière sèche est comprise entre une valeur maximale de $39,25 \%$ notée chez Aegilops triuncialis L. et une valeur minimale de $24,04 \%$ noté chez Plantago lagopus L. La moyenne de la matière sèche est observée chez Papaver rhoeas L., Agropyrum repens L. et Trifolium stellatum $\mathrm{L}$. avec des valeurs respectives de $25 \%$, $36,24 \%$ et $37,50 \%$. Cette différence de la teneur en matière sèche s'explique par la bonne période phénologique dont la quelle les échantillons analysés ont été prélevés. A cette phase végétative l'activité métabolique de la plante étant ralentie, les exigences en eau sont réduites, la transpiration l'emporte sur l'absorption. Le ralentissement de l'activité métabolique et le déséquilibre hydrique entraînent une diminution de la quantité d'eau des plantes. La teneur en matière sèche augmente régulièrement, entre la floraison est le stade vitreux: elle passe de 14-16 à 33-35\% (Demarquilly, 1994). En outre les taux élevés de matière sèche sont également connus comme facteurs limitant de digestibilité des fourrages (Arab, 2009). Ainsi, les cycles biochimiques sont impactés, comme le cycle de l'azote qui s'accélère induisant des changements dans la composition botanique autour des zones de déjection (Gillet et al., 2010).

Tableau 3. Caractéristiques physico-chimiques de l'horizon superficiel du sol des stations étudiées.

Table 3. Physico-chemical characteristics of the surface soil horizon of the stations studied.

\begin{tabular}{lllllll}
\hline & S1 & S2 & S3 & S4 & S5 & S6 \\
\hline Texture & AS & AS & SA & SA & AS & SA \\
Structure & G & G & G & G & Gr & Gr \\
FG (\%) & 31,16 & 14,79 & 17,20 & 45,78 & 35,48 & 45,08 \\
S (\%) & 32,68 & 19,00 & 30,40 & 50,58 & 38,46 & 44,30 \\
A (\%) & 33,26 & 25,70 & 12,70 & 11,47 & 21,91 & 9,13 \\
L (\%) & 4,23 & 6,10 & 4,10 & 4,29 & 6,26 & 6,18 \\
Humidité (\%) & 7,02 & 8,31 & 8,12 & 10,80 & 11,60 & 11,59 \\
pH & 7,60 & 7,42 & 7,23 & 7,56 & 7,42 & 7,52 \\
Conductivité (ms/cm) & 0,36 & 0,10 & 0,10 & 0,06 & 0,09 & 0,08 \\
Matière organique (\%) & 3,75 & 4,00 & 3,50 & 3,68 & 4,05 & 4,42 \\
Calcaire total (\%) & 24,16 & 21,80 & 20,16 & 2,77 & 1,14 & 1,66 \\
Calcaire actif (\%) & 11,5 & 1,22 & 2,13 & 10,37 & 9,00 & 8,75 \\
\hline
\end{tabular}

FG: Fraction Grossière, S: Sable, A: Argile, L: Limon, G: Grenue, Gr: Grumeleuse, As: Argilo-Sableuse, Sa: Sablo-Argileuse.

La teneur en matière organique des espèces étudiées varie entre $86,51 \%$ et $93,45 \%$. Le contenu organique le plus élevé est observé chez Plantago lagopus L. avec une valeur de 93,45 \%. La plus part des espèces étudiées présentent des taux importants en matière organique. Cette faible différence entre les résultats obtenus, peut être liée aux variations des facteurs climatiques, édaphiques, et au stade du développement physiologique des plantes.

Sur l'ensemble des 5 espèces étudiées, la matière minérale présente des teneurs faibles variant d'une espèce à une autre de $6,55 \%$ à 13,49\%. Selon Nedjraoui (1981); la diminution de la matière minérale (cendre) est due à la migration d'azote, de phosphore et de potassium des feuilles, après satisfaction de leurs besoins importants au moment de leurs croissances vers les organes souterrains vivaces ou ils sont mis en réserve.

\section{Teneur en cellulose brute}

La teneur de la cellulose brute varie considérablement d'une espèce à une autre, elle est de $14,85 \%$ à $28,96 \%$. D'une façon globale, les espèces analysées sont riches en cellulose brute,

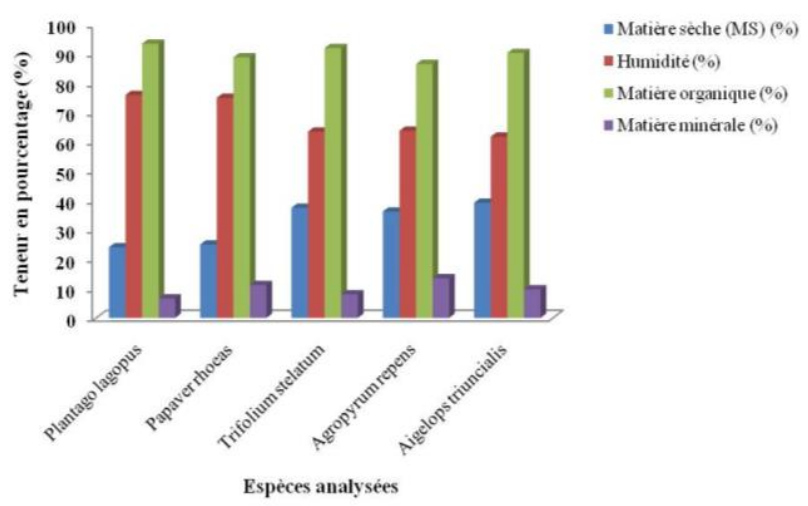

Figure 4. Teneur en matière sèche, humidité, matière organiques et matière minérale en pourcentage (\%) des espèces analysées.

Figure 4. Dry matter, moisture, organic matter and mineral matter content as a percentage (\%) of the species analyzed.

mais toujours nous notons qu'il y a une certaine variabilité interspécifique (Figure 5). Le taux le plus faible est enregistré chez Papaver rhoeas L. (14,85 $\%)$, mais le plus élevé est enregistré chez Trifolium 
stellatum L. $(28,96 \%)$. Cette variation entre les résultats peut être liée au stade de développement de la plante et/ou aux conditions édapho-climatiques des milieux.

\section{Mesure de l'offre fourragère des espèces}

Les résultats obtenus à partir des analyses de l'offre fourragère sont réunis dans le Tableau 4.
Pour prédire la valeur énergétique des plantes, on utilise les équations de prévision proposées par I'INRA (2004). En remplaçant, chacun des paramètres de l'équation par les valeurs moyennes trouvées dans le Tableau 4, les résultats sont répertoriés dans le Tableau 5.

Ces valeurs sont proches aux valeurs indiquées pour différentes fourrages consommés par les ruminants, excepté celle de la Papaver rhoeas L.;

Tableau 4. Valeurs traités après les dosages de l'offre fourragère.

Table 4. Values treated after the feed supply assays.

\begin{tabular}{llllllllll}
\hline Espèces & MS \% & $\begin{array}{l}\text { MO \% } \\
\text { de MS }\end{array}$ & $\begin{array}{l}\text { Kg MO } \\
\text { /Kg MS }\end{array}$ & $\begin{array}{l}\text { Qtité de MS } \\
\text { pour avoir } \\
\text { 1KG MO }\end{array}$ & $\begin{array}{l}\text { CB \% de } \\
\text { MS }\end{array}$ & $\begin{array}{l}\text { Kg } \\
\text { CB/Kg } \\
\text { MS }\end{array}$ & $\begin{array}{l}\% \text { CB/ } \\
\text { Kg MO }\end{array}$ & $\begin{array}{l}\% \\
\text { MAT/Kg } \\
\text { MO }\end{array}$ & MM \% \\
\hline Aegilops triuncialis L. & 39,25 & 90,28 & 0,9028 & 1,11 & 22,53 & 0,2253 & 24,96 & 20,71 & 9,72 \\
Agropyrum repens L. & 36,24 & 86,51 & 0,8651 & 1,16 & 23,64 & 0,2364 & 27,33 & 21,62 & 13,49 \\
Papaver rhoeas L. & 25 & 88,77 & 0,8877 & 1,13 & 14,85 & 0,1485 & 16,72 & 21,07 & 11,23 \\
Plantago lagopus L. & 24,04 & 93,45 & 0,9335 & 1,07 & 18,75 & 0,1875 & 20,09 & 20,03 & 6,55 \\
Trifolium stellatum L. & 37,5 & 91,99 & 0,9199 & 1,09 & 28,96 & 0,2896 & 31,48 & 20,33 & 8,01 \\
\hline
\end{tabular}

MS: matière sèche, MO: matière organique, $\mathrm{CB}$ : cellulose brute, MAT: matière azotée totale, MM: matière minérale.

car elle est très élevée sachant que l'aliment énergétique de référence (l'orge) présente une valeur «U.F.L» égale à 1 (INRA, 1988, 2004). Ceci peut être dû à la forte teneur en matière organique (MO) $(88,77 \%)$ et à la faible teneur en C.B $(14,85 \%$ de M.S) enregistrées chez cette espèce.

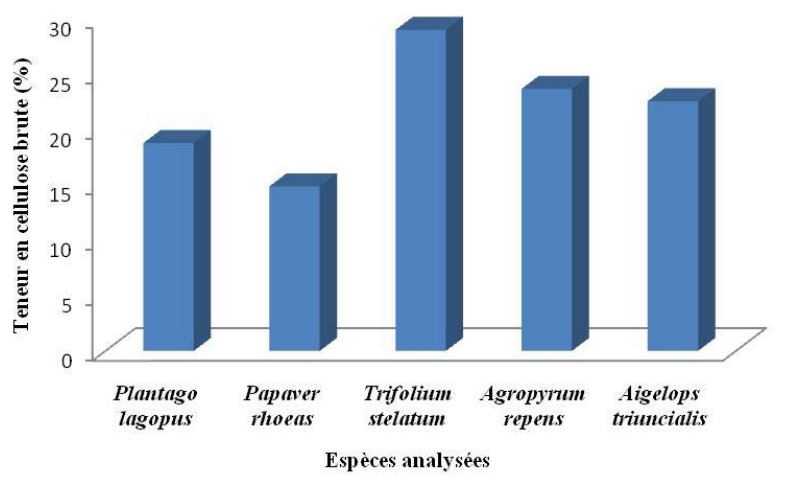

Figure 5. Teneur en cellulose brute en pourcentage (\%) des espèces analysées.

Figure 5. Crude fiber content in percentage (\%) of the species analyzed.

\section{Comparaison entre l'offre et les besoins fourragers du cheptel}

\section{Mesure de la biomasse brute}

La biomasse brute des espèces est mesurée avant l'exécution des analyses de l'offre fourragère, et cette étape était pour rationner l'utilisation de la matière végétale. La quantité fraîche des plantes est représentée dans le Tableau 6.

La quantité moyenne de la biomasse brute répartie dans les six stations échantillonnées des cinq espèces étudiées, diffère d'une espèce à une autre. Cette différence revient à certaines caractéris- tiques relatives à l'espèce elle-même (biologie et physiologie) et aux conditions du milieu. Selon les résultats obtenus, on observe une quantité importante de biomasse brute chez Agropyrum repens $\mathrm{L}$. $(343,6 \mathrm{Kg} / \mathrm{ha})$, par contre elle est faible chez Trifolium stellatum L. (112,3 kg /ha).

Une variation de l'offre fourragère a été enregistrée d'une espèce à l'autre, cette variabilité se traduit par la valeur énergétique (Kcal) ou par l'intérêt fourrager de chacune des espèces. La valeur moyenne de l'offre fourrager fournis par les cinq espèces étudiées est de 154,04 UFL / ha, cette valeur est en parallèle proche à celle trouvée par $\mathrm{El}$ Hamrouni en 1978 (140 UFL / ha) dans une Pineraie en Tunisie sous un étage bioclimatique semi aride inferieur avec une pluviométrie annuelle qui ne dépasse pas $400 \mathrm{~mm}$, l'une des caractéristique de notre zone d'étude.

\section{Calcul des besoins fourragers du cheptel}

Pour cette partie de travail, nous avons voulu évaluer le fourrage fourni au cheptel existant (ovin, bovin, caprin) au moment de l'enquête (mai à juin 2016). On a observé que les ovins se déplacent dans des zones où il y a une végétation claire avec des faibles pentes, il s'agit des Garrigues à base de calycotome (Calicotome spinosa Link.), palmier nain (Chamaerops humilis L.) et diss (Ampelodesmos mauritanicus Poir.) (station S1, S3 et S4). Tandis que les bovins prennent leur nourriture dans des zones caractérisées par une végétation dense telle que les matorrals du versant nord à base de chêne vert (Quercus ilex L.) (S5et S6) et la forêt claire à base de Pin d'Alep (Pinus halepensis Mill.) (S2). Notons ainsi que durant le déroulement de notre enquête, on a remarqué que le nombre important du cheptel exerce un prélèvement excessif de la 
Tableau 5. Les valeurs énergétiques des espèces en Kcal et en UFL.

Table 5. The energy values of the species in Kcal and in UFL.

\begin{tabular}{lllllllll}
\hline Espèces & dMO $\%$ & dE $\%$ & ED Kcal & EM Kcal & q & Km & $\begin{array}{l}\text { UFL } / \mathbf{k g} \\
\text { brut }\end{array}$ & $\begin{array}{l}\text { UFL/kg } \\
\text { MS }\end{array}$ \\
\hline Aegilops triuncialis L. & 68,93 & 66,13 & 2645,35 & 1518,15 & 0,379 & 0,66 & 0,59 & 1,68 \\
Agropyrum repens L. & 67,91 & 64,24 & 2569,70 & 1409,33 & 0,352 & 0,65 & 0,54 & 2,36 \\
Papaver rhoeas L. & 85,18 & 81,23 & 3249,41 & 2495,32 & 0,62 & 0,73 & 1,08 & 3,37 \\
Plantago lagopus L. & 72,41 & 70,19 & 2807,61 & 1751,62 & 0,44 & 0,68 & 0,70 & 2,18 \\
Trifolium stellatum L. & 63,02 & 60,92 & 2436,99 & 1241,53 & 0,31 & 0,64 & 0,47 & 1,46 \\
\hline
\end{tabular}

dMO: digestibilité de la matière organique, dE: digestibilité de l'énergie, ED: énergie digestible Kcal, EM: énergie métabolisable en Kcal, q: concentration en EM de l'aliment: $0<\mathrm{q}<1, \mathrm{Km}$ : efficacité de l'utilisation d'EM en énergie nette (EN) pour l'entretien: comprise entre 0 et 1, UFL: unité fourragère lait= valeur énergétique des plantes pour des ruminants à l'entretien.

végétation. Les besoins fourragères journaliers du cheptel de notre site d'étude sont représentés dans le Tableau 7.

\section{Bilan fourrager}

Dans les stations S1, S3 et S4, l'offre du fourrage fourni par ces espèces est de 11,05 UFL, dont les besoins journaliers du cheptel (525 têtes ovines et caprins) sont de 268,3 UFL. Donc le déficit fourrager est de 8574,55 UFL, ce qui correspond à un manque de $11463,04 \mathrm{Kg}$ de matière sèche. La quantité fourragère fournie par les espèces trouvées dans les stations S2, S5 et S6 est d'une valeur énergétique de 24,28 UFL. Cependant, les besoins journaliers du cheptel existant dans ces stations (66 têtes de bovins) sont estimés à 315 UFL. Alors, le déficit fourrager de la période végétative des espèces est de 10055,72 UFL et qui correspond à un manque de $11512,03 \mathrm{Kg}$ de matière sèche. D'après ces résultats l'offre de la matière sèche pour ces espèces est de $365,56 \mathrm{~kg}$, tandis que les besoins demandés par le cheptel durant la période végétative de ces espèces atteignent 22986,67 kg. II est clair que l'offre fourragère de ces parcours est insuffisante et la charge animale est excessive. Ceci se traduit par une pression de pacage importante sur les plantes et ne leur laisse pas le temps nécessaire pour un renouvellement. Ce déficit est pallié par le pacage en délit dans les ravins et les zones forestières.

Tableau 6. Quantité moyenne de biomasse brute et de matière sèche en UFL pour chaque espèce.

Table 6. Average amount of raw biomass and dry matter in UFL for each species.

\begin{tabular}{lllll|llll} 
& \multicolumn{4}{c}{ Matière brute } & \multicolumn{4}{c}{ Matière sèche } \\
\cline { 2 - 9 } Espèces & $\mathrm{kg} / \mathrm{ha}$ & $\mathrm{UFL} / \mathrm{ha}$ & $\mathrm{kg} / \mathrm{st}$ & $\mathrm{UFL} / \mathrm{st}$ & $\mathrm{Kg} / \mathrm{ha}$ & $\mathrm{UFL} / \mathrm{ha}$ & $\mathrm{Kg} / \mathrm{st}$ & $\mathrm{UFL} / \mathrm{st}$ \\
\hline Aegilops triuncialis L. & 224,75 & 132,6 & 2,25 & 1,33 & 72,82 & 122,33 & 0,72 & 1,22 \\
Agropyrum repens L. & 343,6 & 185,6 & 3,44 & 1,86 & 111,33 & 262,73 & 1,11 & 2,62 \\
Papaver rhoeas L. & 226 & 244,08 & 2,26 & 2,44 & 73,22 & 246,75 & 0,73 & 2,46 \\
Plantago lagopus L. & 221,6 & 155,12 & 2,22 & 1,55 & 71,80 & 156,52 & 0,71 & 1,56 \\
Trifolium stellatum L. & 112,3 & 52,79 & 1,12 & 0,53 & 36,39 & 53,12 & 0,36 & 0,53 \\
\hline
\end{tabular}

\section{Détermination de la charge animale}

Pour la notion de charge, on parle de la charge d'équilibre ainsi que de la charge réelle qui est déterminée par le coefficient d'une unité de gros bétail (UGB).

La charge réelle $(\mathrm{Cr})=$ besoin total du cheptel en UFL divisé par besoins en UFL d'un gros bétail. On a: 1 bovin représente $1 \mathrm{UGB}, 3$ ovins représentent 1 UGB et 4 caprins représentent 1UGB. Pour la période végétative des cinq espèces étudiées: Besoin total du cheptel en UFL $=18665,6$ UFL, besoins en UFL d'un gros bétail = 128 UFL, donc la charge réelle est estimée à 145 UGB. La charge d'équilibre, c'est la charge maximale que peut supporter en moyenne, un pâturage sans que sa flore pastorale ne se dégrade. La charge d'équilibre (Ce): c'est la production fourragère totale en UFL divisée sur les besoins en UFL d'un gros bétail. Dans notre cas d'étude, la production fourragère totale est de 35,33 UFL, les besoins en U F d'un gros bétail avec 128 UFL, ce qui donne une charge d'équilibre de 0,27 UGB ( $\mathrm{Ce}=35,33 / 128=0,27$ UGB). On remarque que la charge réelle est supérieure à la charge d'équilibre ce qui indique une surcharge animale sur les terrains des parcours dans le site d'étude. 
Tableau 7. Besoins fourragères journaliers du cheptel de la zone d'étude.

Table 7. Daily fodder needs of the herd in the study area.

\begin{tabular}{|c|c|c|c|c|c|c|c|}
\hline \multirow[t]{2}{*}{ L'anima } & & \multirow[t]{2}{*}{ Age } & \multirow{2}{*}{$\begin{array}{l}\text { Nombre } \\
\text { des } \\
\text { têtes }\end{array}$} & \multicolumn{2}{|c|}{ Besoins en kg MS } & \multicolumn{2}{|c|}{ Besoins en UFL } \\
\hline & & & & $\begin{array}{l}\mathrm{kg} \mathrm{MS} \mathrm{/} \\
\mathrm{J}\end{array}$ & $\begin{array}{l}\mathrm{kg} \mathrm{MS/} \\
32 \mathrm{~J}\end{array}$ & $\begin{array}{l}\text { UFL / } \\
\text { Jour }\end{array}$ & $\begin{array}{l}\text { UFL / } 32 \\
\text { Jours }\end{array}$ \\
\hline \multirow[t]{2}{*}{ Ovin } & brebis & $>1$ an & 85 & 94,44 & 3022,22 & 37,4 & 1196,8 \\
\hline & agneaux & $<1$ an & 405 & 225,00 & 7200,00 & 210.6 & 6739,2 \\
\hline Caprin & chèvres & $<1$ an & 35 & 38,89 & 1244,44 & 20.3 & 649,6 \\
\hline \multirow[t]{3}{*}{ Bovin } & Génisses & $>1$ an & 15 & 133,33 & 4266,67 & 9 & 288 \\
\hline & Veaux et & $<1$ an & 51 & 226,67 & 7253,33 & 306 & 9792 \\
\hline & & & & Total & 22986,67 & - & 18665,6 \\
\hline
\end{tabular}

Le déficit fourrager s'explique en quelque sorte par une surcharge dans les terrains de parcours dont le coefficient est donné parle coefficient de surpâturage (S), il s'agit du rapport de la charge d'équilibre à la charge réelle. II est exprimé en pourcentage de la charge d'équilibre. La relation peut s'écrire: $S=100(1-\mathrm{Ce} / \mathrm{Cr})$. Dans notre cas: $\mathrm{Ce}=0,27$ UGB; $\mathrm{Cr}=145 \mathrm{UGB}$, le coefficient de surpâturage est estimé à $99 \%(S=(1-0,27)$ 145)100) ce qui signifie un excès de charge. Cela veut dire, que le disponible fourrager des cinq espèces réparties dans les six stations est insuffisant aux besoins du cheptel. II est utile de remarquer que ce coefficient est plus important que celui trouvé par El Hamrouni en 1992 et qui est de $77 \%$. Ceci peut être expliqué par le fait que la surface utilisée pour cette étude ne peut pas indiquer clairement le surpâturage surtout lorsqu'il s'agit d'un écosystème plus diversifié et d'un parcours assez vaste, comme le cas du mont de Tessala, dont plus d'espèces contribuent dans l'alimentation du cheptel. Le déficit fourrager et le fort coefficient de surpâturage calculés ne peuvent que donner un aperçu sur l'état actuel du parcours au niveau du mont de Tessala et ils confirment cependant l'intensité de la pression animale sur l'écosystème étudié.

\section{Analyse statistique}

L'analyse factorielle des correspondances (AFC) a permis d'identifier deux groupes de formations végétales (Figure 6). L'axe F1 (60,80\% d'inertie) exprime un gradient d'évolution des habitats équilibrés vers les stations dégradées et fortement pâturées. La classification hiérarchique ascendante distingue nettement l'individualisation de ces 2 groupes.

Le groupe Gr 1: c'est un groupe représenté par les stations S2, S5 et S6 ou domine les types biologiques: phanérophytes et nano phanérophytes. Ce groupe se voie rattaché aux conditions du milieu les plus évoluées tel que: la matière organique (MO), le taux des limons $(\mathrm{L})$, le taux de recouvrement de la végétation (Rct) et le facteur altitude (Alt). Ce groupe caractérise les matorrals arborés et denses, situés en exposition Nord avec un sol limoneux et parfois sableux riche en matière organique, ou domine le chêne vert (Quercus ilex) (S5 et S6) en association avec les espèces suivantes: Quercus coccifera L.,
Arbutus unedo L., Viburnum tinus L., Pistacia lentiscus L., Lonicera implexa Aiton., Rosa sempervirens L., Crateagus monogyna Jacq., Ampelodesmos mauritanicus Poir., Daphne gnidium L., Phillyrea angustifolia L. et Cistus salviifolius L., et la forêt claire à base de Pinus halepensis Mill. (S6) situé en exposition sud, le taux de recouvrement de ces formations végétales varie de $30 \%$ à $35 \%$ dont leur densité importante les protège du pâturage.

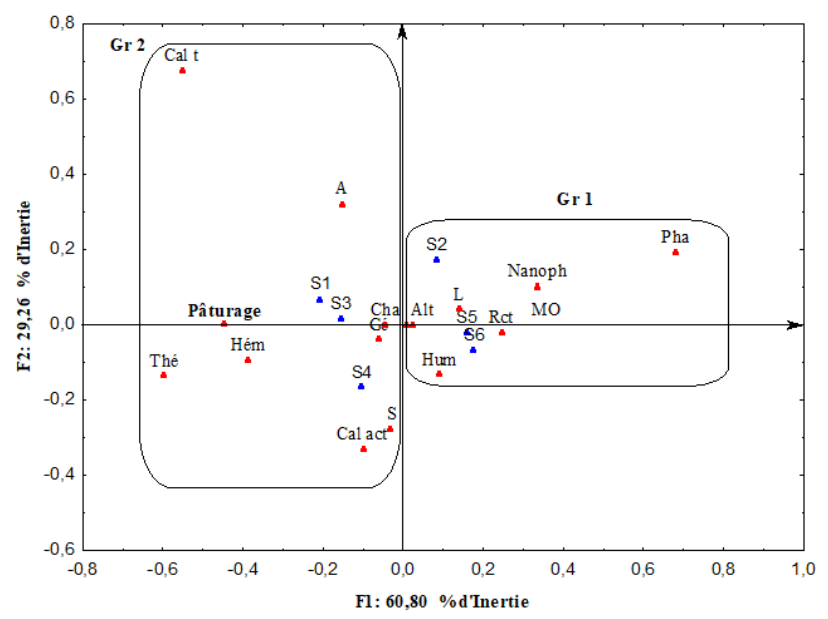

Figure 6. Représentation graphique du plan factoriel F1xF2. Légende: S: Sables, SF, L: Limons, A: Argiles, MO: Matière organique, Cal $t$ : calcaire total, Hum: Humidité du sol, Alt: Altitude, Ph: Phanérophytes, Nanoph: Nanopahnéophytes, Ch: Chaméphytes, Ge: Géophytes, He: Hémicryptophytes, Rct: Recouvrements de la végétation, S: Stations.

Figure 6. Graphic representation of the factorial plane F1XF2.

Le groupe $\mathrm{Gr} 2$ : c'est un groupe représenté par les stations $\mathrm{S} 1, \mathrm{~S} 3$ et $\mathrm{S} 4$. Ces dernières sont représentées par les types biolologiques; chamephytes, géophytes, hémicryptophytes et les thérophytes. Ce groupe est corrélé aux paramètres suivants: sables (S), argile (A), le taux de calcaire (Cal act, Cal t) et le facteur pâturage. Ce groupe est caractérisé par des stations assez dégradées ou domine les espèces suivantes notamment: Calicotome spinosa Link., Chamaerops humilis L., Asparagus acutifolius L., Asphodelus microcarpus Sal et Viv., Malva sylvestris L., Marrubium vulgare L., Ruta montana L., Stipa tenassicima L., Bromus rubens L., Thymus ciliatus Desf., et Urginea maritima L.. Les stations citées sont caractérisées par un sol fortement calcaire et pauvre en matière organique ce qui favorise l'installation de ces peuplements témoignant d'une dégradation dont l'origine est l'effet anthropique exercé par l'homme et son troupeau d'une part et l'agressivité du climat d'autre part (Cherifi et al., 2011, 2014, 2017). La charge animale est excessive traduisant une pression de pacage sur les plantes consommées notamment les cinq espèces étudiées (Aegilops triuncialis L., Agropyrum repens L., Papaver rhoeas L., Plantago lagopus L. et Trifolium stellatum L.) et ne laisse pas le temps nécessaire pour leur renouvellement. Nous notons ainsi la présence des espèces indicatrices de 
mauvaise gestion pastorale, voire de surpâturage, ont été recensées dans les stations subissant une forte pression pastorale (S1, S3 et S4). II s'agissait d'espèces classées dans la catégorie des refus, comme Centaurea calcitrapa L., Centaurea incana Desf., Dactylis glomerata L., Echinops spinosus L., Erodium moschatum (L.) L'Hér., Ruta montana L., Ruta chalepensis L., Scolymus hispanicus L., qui se multipliaient au détriment des espèces bonnes fourragères, lesquelles, au contraire, avaient tendance à régresser sous l'effet de la sélection par le bétail.

\section{Conclusion}

A travers cette étude a été mis en évidence l'impact de l'élevage pastoral extensif et traditionnel sur les écosystèmes naturels. L'état de l'écosystème forestier du Mont de Tessala est en dégradation, l'analyse des facteurs actuels montre l'ampleur de l'influence de surpâturage sur cet écosystème qui reste sans contexte parmi les éléments déterminants de la régression du tapis végétal.

Dans notre cas d'étude, l'analyse fourrager est considéré comme étant un facteur directeur dans le diagnostic de l'impact de surpâturage sur la dynamique de la végétation, aussi en prenant en compte la contribution de l'indicateur floristique dans la distinction entre les formations végétales affectés par le pâturage à travers la richesse spécifique des espèces dans chaque formation végétale ou station d'échantillonnage.

Les résultats de l'analyse fourragère ont permis de déterminer, sur le plan qualitatif, un bilan fourrager entre les besoins énergétiques du cheptel (en UFL) existant dans le site d'étude (490 têtes d'ovins, 35 têtes des caprins et 66 têtes des bovins) et la quantité de l'énergie que nos espèces étudiées offrent pendant la période végétative (du mois d'Avril à mois de Juin). Ce bilan fourrager se traduit par un déficit remarquable dans les deux versants, néanmoins le versant nord présente un déficit peu important (11512,03 Kg de matière sèche) que celui présenté dans le versant sud $(11463,04 \mathrm{Kg}$ de matière sèche) car la consommation diffère selon le type de l'espèce animale.

Les résultats obtenus à la suite de l'étude et l'analyse des données sylvo-pastorales dans la strate herbacée, ainsi que le bilan fourrager actuel et le fort coefficient de surpâturage estimé à $99 \%$ ne peuvent nous informer que sur une pression du pâturage au quelle est soumis l'écosystème forestier du Mont de Tessala, phénomène qui pourra, sans doute s'il se continue, de perturber tout un écosystème.

Il est donc nécessaire d'avancer des propositions de réhabilitation des potentialités pastorales dégradées. Ces propositions doivent tenir compte du contexte écologique de la région, des aspirations de la population rurale habitant le milieu forestier. Ainsi, on doit chercher à faire participer cette population dans la prise des décisions surtout quand il s'agit de toucher la seule source de revenu qui est le cheptel. Les propositions doivent considérer entre autre la réalité économique du pays et les stratégies régionales déjà tracées.

\section{Références bibliographiques}

APG III. (2009). An update of the Angiosperm Phylogeny Group classification for the orders and families of flowering plants: APG II. Botanical Journal of the Linnean Society, 161,105-121.

Arab, H., Haddi, M.L. \& Mehennaoui, S. (2009). Evaluation de la valeur nutritive par la composition chimique des Principaux fourrages des zones aride et semi-aride en Algérie. Sciences \& Technologie, 30, 50-58.

Baraka, D. (2008). Inventaire et caractérisation des plantes médicinales de djebel Tessala (wilaya de Sidi Bel Abbes). Mémoire de magister en biologie. Université djilali liabès de Sidi Bel Abbes, Algérie. 100 p.

Barbero, M., Bonin, G., Loisel, R. \& Quézel, P. (1990). Changes and disturbances of forest ecosystems caused by human activities in the western part of Mediterranean bassin. Vegetatio, 87, 151-173.

Benabdeli, K. (2000). Evaluation de l'impact des nouveaux modes d'élevage sur l'espace et l'environnement steppique: Cas de Ras El Ma (Sidi Bel Abbes - Algérie). In: Rupture: Nouveaux enjeux, nouvelles fonctions, nouvelle image de l'élevage sur parcours.

Options Méditerranéennes, 39, 129-141.

Benzécri, JP. (1984a). L'analyse des données: la taxonomie. vol. 1, 635 p. Éd. Dunod.

Benzécri, JP. (1984b). L'analyse des correspondances. vol. 2, 632 p., Éd. Dunod.

Bonin, G. \& Tatoni, T. (1990). Réflexions sur apport de l'analyse factorielle des correspondances dans l'étude des communiés végétales et leur environnement. Volume jubilaire du Prof. Quézel. Ecologia Mediterannea, 16, 403-414.

Bouazza, M., Benabadji, N., Loisel, R. \& Metge, G. (2004). Evolution de la végétation steppique dans le Sud-Ouest de l'Oranie (Algérie). Ecologia Mediterranea, 30, 219-31.

Bouterfas, K. (2011). Étude de Marrubium vulgare L. du mont de Tessala (Algérie occidentale): autoécologie, histologie, quantification de quelques polyphénols et évaluation du pouvoir antimicrobien des flavonoïdes. Thèse de magister. Université Djillali Liabès de Sidi Bel Abbès (Algérie).

Bouzidi, M., Latreche, A., Attaoui, I., Benabderrahmane, M., Mehdadi, Z. \& Benyahia, M. (2012). Antibacterial Effect of the Essential Oils Extracted From Ruta chalepensis L. and Ruta montana (L.) L. Journal of Life Sciences, 6 (8), 898-902.

Braun-Blanquet, J. (1951). Pflanzensoziologie Grundzuge der vegetations Kunde (2 ${ }^{\mathrm{ème}}$ ed.) Spring. Vienne, Autrich, pp 631. 
Cherifi, K. (2013). Impact de l'action anthropozoogéne sur la biodiversité végétale de l'écosystéme forestier du Djebel Tassala (Algérie occidentale). Thèse Doct. Eco. Végétale et environnement. Univ. Sidi Bel Abbes. 100p+ annexes.

Cherifi, K., Mehdadi, Z. Et Bachir Bouidjra, S. (2011). Impact de l'action anthropozoogène sur l'écosystème forestier du mont de Tessala (Algérie occidentale). Sécheresse, 22, 197-206.

Cherifi, K., Mehdadi, Z., Elkhiati, N., Latreche, A. et Ramdni, M. (2017). Floristic composition of the mountainous massif of Tessala (Algerian West): Biodiversity and regressive dynamics of the forest ecosystem. J. Mater. Environ. Sci, 8(9), 31843191.

Cherifi, K., Mehdadi, Z., Latreche, A., Hazem, Z. \& Elzerey, W. (2014). Impact of livestock grazing on the floristic composition: a case study of the Mount of Tessala, Western Algeria. International Journal of Environnnement, 3(3), 2091-2854.

Clarke, K.R. \& Warwick, R.M. (2001). A further biodiversity index applicable to species lists: variation in taxonomic distinctness. Marine Ecology Progress, pp 265-278.

Daget, Ph. \& Poissonet, J. (1997). Biodiversité et végétation pastorale. Rev. Élev. Méd. Vét. Pays Trop, 50, 141-149.

Demarquilly, C. (1994). Facteur de variation de la valeur nutritive du mais ensilage INRA. Prod, Anim, 7(3), 177-189.

Djebaili, S. (1984). Steppe algérienne, phytosociologie et écologie. Recherche phytosociologique et écologique sur la végétation des hautes plaines steppiques et de l'Atlas saharien. Alger: Office des publications universitaires (OPU).

El Hamrouni, A. (1978). Etude phyto-ecologique et problèmes d'utilisation de l'aménagement dans les forets de Pin d'Alep de la region de Kassarine (Tunisie centrale). Thèse Doct. $3^{\text {ème }}$ cycle. Univ. Aix-Marseille III.

El Hamrouni, A. (1992). Vegetation forestiere et preforestiere de la Tunisie. Typologie et elements pour la gestion. Thèse d'Etat. Univ. Aix-Marseille III. pp. 19-220.

Emberger, L. (1971). Travaux de botanique et d'écologie, Masson, Paris, 520 p.

Gaouar, A. (1980). Hypothèses et réflexions sur la dégradation des écosystèmes forestiers dans la région de Tlemcen. Forêt Méditerranéenne, 2, 131-46.

Gillet, F., Kohler, F., Vandenberghe, C. \& Buttler, A. (2010). Effect of dung deposition on smallscale patch structure and seasonal vegetation dynamics in mountain pastures. Agriculture. Ecosystems and Environment, 135, 34-41.

Gounot, M. (1969). Méthodes d'études quantitatives de la végétation. Éditions Masson et Cie. Paris. $314 \mathrm{p}$.

Hakemi, Z., Mehdadi, Z., Latreche, A., Cherifi, K. \& Aisset, A. (2018). Habitat characterization of Aristolochia baetica L. in Tessala mount, western Algeria. Rev. Biodiversitas. 19 (5), 1633-1641.

INRA. (1988). Alimentation des bovins ovins et caprins. INRA Editions. Paris. $471 \mathrm{p}$.

INRA. (2004). Tables de composition et de valeur nutritive des matières premières destinées aux animaux d'élevage: porc. In Sauvant D., Perez J.M., Tran G. (eds), 2ème Edition revue et corrigée. INRA Editions. Paris. 301 p.

Kadi-Hanifi, H. (2003). Diversité biologique et phytogéographique des formations à Stipa tenacissima L. de l'Algérie. Sècheresse, 14, 169179.

Kiekken, R. (1962). Géologie et stratigraphie des monts du Tessala. Ed. Fouquet. Oran. 220p.

Mokaddem, M. (2012). Comparaison entre les formations végétales de Quercus ilex L. et Pinus halepensis Mill. et valorisation de l'espèce Rubus ulmifolius Schott. Mémoire de magister, Université DjillaliLiabès de Sidi Bel Abbès, Algérie. $116 \mathrm{p}$.

Nedjraoui, D. (1981). Evolution des éléments biogènes et valeurs nutritives dans les principaux faciès de végétation (Artemisia herba-alba, Lygeum spartum et Stipa tenacissima) des Hautes Plaines steppiques de la Wilaya de Saïda. Thèse de 3ème cycle. USTHB, Alger. $156 \mathrm{p}$.

Ouici, H., Mehdadi, Z., Cherifi, K. \& El ZereyBelaskri, A. (2015). Inventory and Analysis of Phytodiversity along an Altitudinal Gradient in the Southern Slope of the Mount of Tessala (Western Algeria). Open Journal of Ecology, 5, 552-562.

Ozenda, P. (1982). Les végétaux dans la biosphère. Éditions Doin. Paris, $432 \mathrm{p}$.

Quézel, P. \& Santa, S. (1962). Nouvelles flores de l'Algérie et des régions méridionales. Paris. Edition CNRS. Tome I. 565 p.

Quézel, P. \& Santa, S. (1963). Nouvelle flore de l'Algérie et des régions désertiques \& méridionales. Paris. Edition CNRS. Tome II. 605 p.

Quézel, P. (2000). Réflexion sur l'évolution de la flore et de la végétation au Maghreb Méditerranéen. Ibis. Press. Edit. Paris. $117 \mathrm{P}$.

Sauvage, CH. (1961). Recherches géobotaniques sur le chêne liège au Maroc. Thèse Doct. Etat, Montpellier, Trav. Inst. Sci. Chérifien, Série Botanique, pp. 21- 462 . 